\title{
Reconsidering Approaches to Estimating Health Disparities Across Multiple Measures of Sexual Orientation
}

\author{
Jessica N. Fish, $\mathrm{PhD}^{1}$ and Evan A. Krueger, PhD, MPH, MSW²
}

\begin{abstract}
Purpose: We propose a new theoretically grounded approach for estimating sexual orientation-related health risk that accounts for the unique and shared variance of sexual identity across other measures of sexual orientation (i.e., attraction and behavior). We argue and illustrate that this approach provides specificity not demonstrated by approaches that independently estimate and compare health risk based on sexual identity, attraction, and behavior.

Methods: Data were from the National Epidemiologic Survey on Alcohol and Related Conditions-III, collected in 2012-2013 ( $N=36,309$, ages 18 and older). The Karlson-Holm-Breen method tested the degree to which attraction- and behavior-based disparities in mental health and substance use disorders change after adjusting for sexual identity.

Results: Sexual attraction- and behavior-based disparities in mental health and substance use disorders statistically varied when comparing models that did and did not adjust for sexual identity. Adjusting for sexual identity appeared to have a larger influence on attraction- and behavior-based health associations among men; sexual minority and majority differences were attenuated on nearly every outcome after adjusting for sexual identity. This attenuation was less common among women. Among women, some behavior-based disparities were wider in sexual identity-adjusted models relative to unadjusted models.

Conclusion: We demonstrate more accurate approaches to capturing and comparing sexual orientation-related health disparities across multiple measures of sexual orientation, which account for the shared variance between sexual identity and measures of attraction and behavior. Adjusted estimates provide more specificity regarding relative health risk across specific subgroups of sexual minority people, and the intervention and prevention strategies needed to address them.
\end{abstract}

Keywords: health disparities, LGB, mental health, sexual minority, substance use

\section{Introduction}

Qexual minority (SM) PEOPLE evidence stark health $\checkmark$ inequities when compared with their heterosexual counterparts. ${ }^{1-4}$ Recognizing the heterogeneity across self-reported sexual identity, attraction, and behavior, ${ }^{5-7}$ researchers have capitalized on the inclusion of multiple measures of sexual orientation in large, population-based samples to document and compare mental health and substance use disparities between SM and sexual majority adults based on sexual identity, behavior, and attraction independent of one another. ${ }^{8-10}$ This approach often yields results that highlight differential risk for SM people depending on the measure of sexual orientation used to estimate differences. ${ }^{8-11}$ We argue, however, that there are more accurate approaches to capture and compare differences in sexual orientation-related health disparities across multiple measures of sexual orientation, which account for the shared variance between sexual identity and measures of attraction and behavior.

\section{Estimating health disparities across multiple measures of sexual orientation}

Several population-based studies now incorporate two or more measures of sexual orientation, which include items assessing participants' sexual and/or romantic attraction, behavior, and identity (e.g., the National Epidemiologic Survey on Alcohol and Related Conditions, the National Longitudinal Study of Adolescent to Adult Health, and the Youth Risk Behavior Surveillance System, among others). Given the availability of multiple sexual orientation measures, it has

\footnotetext{
${ }^{1}$ Department of Family Science, School of Public Health, University of Maryland, College Park, Maryland, USA.

${ }^{2}$ Department of Preventive Medicine, Keck School of Medicine, University of Southern California, Los Angeles, California, USA.
} 
become increasingly common for researchers to assess and compare how these different aspects of sexual orientation are independently associated with health-in other words, to model the association between sexual orientation and health across measures of identity, attraction, and behavior, independent of one another. ${ }^{8-10}$

Indeed, studies that assess sexual orientation differences in health demonstrate differential risk for SM youth and adults across different measures of sexual orientation. ${ }^{8,10-12}$ For example, in their examination of mood and anxiety disorders, Bostwick et al. ${ }^{8}$ found that, based on identity, lesbian women had 1.5 times greater odds, and bisexual women 2.6 times greater odds, of a lifetime mood disorder than heterosexual women. Comparatively, they found no differences in rates of lifetime mood disorders between women exclusively attracted to men, and women who were exclusively attracted to women, mostly women, or equally to both men and women. This approach to modeling and comparing relative health risk across measures of sexual orientation has been a useful strategy to examine the aspect(s) of sexuality that are hypothesized to be most relevant to the health factor of interest (e.g., sexual behavior to assess risk for sexually transmitted infections/HIV), ${ }^{1}$ and have provided vital information on the heterogeneity of sexual orientation-related health risk.

We contend, however, that this approach inadvertently produces estimates that lack specificity for two interrelated reasons. First, the majority of lesbian, gay, and bisexual (LGB) people report same-sex attraction and/or behavior, ${ }^{1}$ and so attraction- and behavior-based models of health risk are heavily influenced by the association between sexual identity and health. That is, attraction- and behavior-based models would include most, if not all, LGB-identified people within a given sample-which likely alters the degree to which attraction and behavior are associated with health. Second, and conversely, many people who report same-sex attraction and/or behavior do not identify as sexual minorities (e.g., LGB), particularly during adolescence and young adulthood. $^{13-16, *}$ As such, models that do not adjust for sexual identity when estimating associations between attraction and/or behavior and any given health outcome do not properly estimate health risk for heterosexual-identified SM people; for example, those with a heterosexual identity but same-sex attraction and/or same-sex behavior, the combination of which influences their day-to-day experiences, behaviors, and subsequently health. ${ }^{15-19}$

In other words, not accounting for the unique and shared variance of sexual identity alongside other measures of sexual orientation obscures health differences across measures of attraction and behavior independent of that sexual identity. These two interrelated issues demonstrate how the approach to independently estimating sexual orientation differences in health risk across discrete measures of sexual orientation lacks specificity and limits the utility of the results drawn from this approach to make strong inferences about differential risk based on sexual identity, attraction,

\footnotetext{
*These two points are further demonstrated by our findings in Figure 1 (see Results section) where we present the overlap between dimensions of sexual orientation (i.e., identity, attraction, and behavior) for SM respondents (i.e., those endorsing at least one non-heterosexual aspect of orientation) in the nationally representative sample used for this study.
}

and behavior. The rationale for why sexual identity is a unique but interrelated pathway to health is supported by the existing theoretical and theoretically grounded empirical literature. $^{15,18,20,21}$

\section{How sexual identity influences attraction- and behavior-based disparities}

The minority stress framework ${ }^{22-24}$ is a guiding explanatory model for the study of sexual orientation-related health disparities. Core tenets of this framework posit that SM people experience compromised health due to exposure to social stressors related to their minority statuses, such as stigma, discrimination, threats of violence, and rejection. Importantly, minority stress theory can be applied broadly-including to those who have same-sex attractions or samesex behaviors independent of a non-heterosexual identitygiven that the absence of LGB identification does not inherently preclude exposure to anti-LGB stigma considering the pervasiveness of heteronormativity in the day-to-day social context. $^{22,23,25}$

However, SM people with same-sex attractions and/or behaviors who also adopt and assert a (stigmatized) LGB identity are uniquely vulnerable to enacted forms of stigma, ${ }^{17,18,20,21}$ likely due to the public and visible nature of social identity. In other words, sexual identity may mediate, moderate, or confound ${ }^{\dagger}$ the degree to which attraction and behavior are related to health. Indeed, emerging research suggests that LGB-identified SM people are more likely to experience enacted stigma, such as discrimination ${ }^{19,21,26}$ and that associations between discrimination and compromised health are stronger for those who identify as LGB, relative to those who report same-sex attraction and behavior, but not a SM identity. ${ }^{18,19}$ For example, young people who report being "out" are more susceptible to family rejection and school-based victimization, ${ }^{27,28}$ which are associated with compromised mental health and substance use among SM youth and adults. ${ }^{11,29,30}$

At the same time, there are unique features of same-sex attraction and same-sex behavior that contribute to health risk, regardless of whether or not people identify as LGB or heterosexual. HIV risk, for example, is more closely associated with sexual behavior than with sexual identity, and so HIV epidemiology research has relied heavily on samples of men who have sex with men (MSM), many of whom identify as heterosexual. However, important differences in HIV risk and prevention behaviors also exist based on sexual identity among MSM, ${ }^{31-33}$ which may be related to internalized stigma and community resources. ${ }^{34}$ Watson et al., ${ }^{33}$ for instance, found that latent profiles characterized mostly by bisexual MSM, but also heterosexual and gay men, most of whom were "closeted" were less likely than a latent profile largely characterized by gay and bisexual men who were "out" to engage in HIV testing and had less knowledge about pre-exposure prophylaxis (PrEP) and postexposure prophylaxis (PEP). They argued that identity-based community

\footnotetext{
${ }^{\dagger}$ Given that there are many processes by which sexual identity can affect health and associations among attraction, behavior, and health, we henceforth use the terms "alter," "influence," and "change" to articulate how sexual identity may modify estimates of attractionand behavior-based differences in health.
} 
connection may facilitate HIV testing and access to PrE$\mathrm{P} / \mathrm{PEP}$ information. Altogether, these points illustrate that SM identity, or lack thereof, plays a unique role in driving or altering stress-related experiences and access to healthpromoting resources for SM people.

\section{The current study}

We use nationally representative data to demonstrate the utility of a new approach to estimating SM health inequities across multiple measures of sexual orientation. We do this by comparing estimates of sexual attraction- and behaviorbased disparities in past-year alcohol use disorder, tobacco use disorder, major depressive disorder, and suicide attempt in models that first exclude (unconditional) and then include (conditional) sexual identity as a covariate. We stratify analyses by sex, given well-established differences in sexual orientation-related health disparities based on sex. This approach also allows us to assess whether there are sex differences in the degree to which attraction- and behavior-based health disparities change when adjusting for sexual identity. The results reflect the association between sexual attraction and/or behavior adjusted for the unique effect of identity on health.

\section{Methods}

\section{Data sources and analytic sample}

Data are from the National Epidemiologic Survey on Alcohol and Related Conditions-III (NESARC-III). Collected in 2012-2013, the NESARC-III is a nationally representative, in-home, cross-sectional survey of noninstitutionalized civilian adults in the United States (ages 18 and older, $N=36,309) .{ }^{35}$ Participants completed the Alcohol Use Disorder and Associated Disabilities Interview Schedule 5, which collected information on Diagnostic and Statistical Manual of Mental Disorders, Fifth Edition (DSM-5) ${ }^{36}$ diagnostic criteria for several mood, anxiety, and substance use disorders through computer-assisted personal interviews. Extensive details regarding the survey methodology have been published previously. ${ }^{35}$ For the current study, we included all available participants who had valid sexual orientation measures $(n=34,803)$. The sample characteristics are presented in Table 1.

Table 1. Sample Characteristics

\begin{tabular}{|c|c|c|c|c|}
\hline & Total & Men & Women & $\mathrm{p}$ \\
\hline$N\left(\%_{\mathrm{w}}\right)$ & $34,803(100.00)$ & $15,237(48.23)$ & $19,566(51.77)$ & \\
\hline Sexual attraction & & & & $<0.001$ \\
\hline Exclusively same-sex & 2.69 & 2.81 & 2.58 & \\
\hline Men and women & 5.21 & 3.80 & 6.52 & \\
\hline Exclusively other-sex & 92.10 & 93.40 & 90.89 & \\
\hline Sexual behavior & & & & $<0.001$ \\
\hline Exclusively same-sex & 2.26 & 2.90 & 1.67 & \\
\hline Men and women & 3.50 & 2.36 & 4.57 & \\
\hline Exclusively other-sex & 94.23 & 94.74 & 93.76 & \\
\hline Sexual identity & & & & 0.039 \\
\hline LGB & 2.80 & 2.57 & 3.02 & \\
\hline Heterosexual & 97.20 & 97.43 & 96.98 & \\
\hline Age (mean) & 47.08 & 46.42 & 47.70 & $<0.001$ \\
\hline Race/ethnicity & & & & $<0.001$ \\
\hline White & 66.51 & 66.97 & 66.08 & \\
\hline Black & 11.8 & 11.09 & 12.47 & \\
\hline $\mathrm{AI} / \mathrm{AN}$ & 1.56 & 1.33 & 1.78 & \\
\hline API/Hawaiian & 5.46 & 5.44 & 5.47 & \\
\hline Hispanic & 14.68 & 15.17 & 14.21 & \\
\hline Born in United States & & & & 0.047 \\
\hline Yes & 84.15 & 83.71 & 84.55 & \\
\hline No & 15.85 & 16.29 & 15.45 & \\
\hline Education & & & & $<0.001$ \\
\hline$<$ High school & 12.83 & 13.20 & 12.48 & \\
\hline High school & 25.71 & 26.76 & 24.72 & \\
\hline Some college & 33.00 & 31.11 & 34.77 & \\
\hline Bachelor's degree & 14.00 & 13.90 & 14.10 & \\
\hline$>$ Bachelor's degree & 14.46 & 15.03 & 13.93 & \\
\hline Household income & & & & $<0.001$ \\
\hline$<\$ 25,000$ & 26.77 & 23.46 & 29.85 & \\
\hline$\$ 25,000-49,999$ & 25.85 & 25.55 & 26.13 & \\
\hline$\$ 50,000-79,999$ & 19.79 & 20.79 & 18.86 & \\
\hline$\$ 80,000-99,999$ & 8.75 & 8.86 & 8.65 & \\
\hline$\$ 100,000+$ & 18.84 & 21.33 & 16.51 & \\
\hline
\end{tabular}

Differences between men and women were tested with design-adjusted $F$ (categorical variables) and Wald tests (continuous variables). AI/AN, American Indian/Alaska Native; API, Asian/Pacific Islander; LGB, lesbian, gay, and bisexual. 


\section{Measures}

Mental health and substance use. Respondents were classified as meeting criteria for DSM-5 mental health and substance use disorders. We included two such measures of substance abuse (past-year alcohol use disorder and pastyear tobacco use disorder) and one measure of mental health (past-year major depressive disorder). Symptoms of each disorder were assessed according to DSM-5 criteria. ${ }^{36}$ For substance use disorders, the threshold was two or more symptoms in the past year; categorization for major depressive disorder was based on a clinical significance criterion. We also assessed whether respondents had attempted suicide in the past year, which was calculated by subtracting age at most recent attempt from the respondent's current age. There were no missing data for mental health and substance abuse outcomes.

Sexual identity. Respondents were asked to choose the "category that best describes your sexual orientation." Response options were "heterosexual (straight)," "gay or lesbian," "bisexual," "not sure," and "unknown." Respondents were categorized as heterosexual ("heterosexual [straight]") or LGB ("gay or lesbian," "bisexual"). Those selecting "not sure" $(n=199)$ or "unknown" ( $n=314)$ were set to missing.

Sexual attraction. Respondents were asked to report the "best description of your sexual attraction to other people." Response options were "'only attracted to females," " attracted to females," "equally attracted to females and males," "mostly attracted to males," "only attracted to males," and "unknown." Using self-reported sex, respondents were categorized as having exclusively other-sex attraction (e.g., for males, "only attracted to females"), exclusively same-sex attraction (e.g., for males, "only attracted to males") or attractions to both men and women (e.g., for males, "mostly attracted to females," "equally attracted to females and males," and "mostly attracted to males"). Those selecting "unknown" were set to missing $(n=335)$.

Sexual behavior. Respondents were asked to report the "gender of sexual partners in [your] entire life." Response options were "only males," "only females," "both males and females," "unknown," and "never had sex." By referencing self-reported sex, respondents were categorized as having exclusively other-sex sexual behaviors, exclusively same-sex sexual behaviors, and sexual behaviors with men and women. Those selecting "unknown" $(n=365)$ or "never had sex" $(n=908)$ were set to missing."

Sex. Participants were coded as either "male" or "female.",

Covariates. All models were adjusted for age (in years), race/ethnicity (White, Black/African American, American Indian/Alaska Native, Asian/Pacific Islander/Hawaiian, His-

\footnotetext{
*We exclude those who report never having sex to help simplify our empirical example of the theoretical arguments we are making here. The association between sexual behavior and health for this group would theoretically be influenced by identity, particularly in studies among youth.
}

panic), nativity (U.S.-born or not), level of completed education (survey response options collapsed as follows: less than high school, high school, some college, college graduate, professional degree), and household income (survey response options collapsed as follows: $<\$ 25,000$, $\$ 25,000$ $\$ 49,000, \quad \$ 50,000-\$ 79,999, \quad \$ 80,000-\$ 99,999$, and $\$ 100,000$ or more). Missing values for covariates were imputed by the NESARC study team using the "assignment method," when the true value could be inferred from other information in the screener or interview or the "hot deck" procedure, in which missing values were replaced with a value from a similar, randomly chosen respondent in the sample.

\section{Analytic approach}

We illustrate the potential of sexual identity to alter attraction- and behavior-based differences in health using the Karlson-Holm-Breen (KHB) approach for comparing coefficients of nested nonlinear probability models. ${ }^{37-39}$ Specifically, we estimated the main effects of attraction and behavior on each health-related outcome (unconditional models), adjusted effects that account for the unique variance explained by sexual identity (conditional model), and the statistical differences of the effect between the two models. Unlike nested logistic regression models, KHB models are not impacted by the rescaling that occurs when additional covariates (e.g., identity) are added to the model. ${ }^{37}$ We elected to use the KHB approach for these reasons, but future disparities research can continue to rely upon "classic" approaches (e.g., linear and logistic regressions) to estimate health risk unless it is necessary to compare coefficients across nonlinear models. All analyses were weighted to the U.S. adult population. ${ }^{35}$ Although standard approaches to clustering could not be applied with the KHB method, we used a cluster sandwich estimator to adjust standard errors to account for potential intragroup correlations.

\section{Results}

For clarity on the rationale of our conceptual approach to modeling sexual orientation-related disparities across measures of sexual orientation, we present the overlap between sexual identity, attraction, and behavior among respondents who endorsed at least one nonheterosexual aspect of sexual orientation (Fig. 1). Select comparisons are discussed herein. Among men, $29.96 \%$ reported a gay or biseuxal identity, reported same-sex attraction, and also engaged in same-sex behavior in their lifetime. An additional 24.04\% reported same-sex attractions and lifetime behaviors but reported a heterosexual identity. Nearly $30 \%$ reported same-sex attractions in the absence of same-sex behaviors or an LGB identity, and $13.23 \%$ reported same-sex behaviors in the absence of same-sex attractions or an LGB identity. Similarly, among women, $24.85 \%$ reported an LGB identity, reported samesex attraction, and also engaged in same-sex behaviors, whereas $21.05 \%$ reported same-sex attractions and behaviors plus a heterosexual identity. Over a third (37.77\%) of women reported same-sex attractions in the absence of same-sex behaviors or an LGB identity, and $12.50 \%$ reported same-sex behaviors in the absence of same-sex attractions or an LGB identity. Less than $1 \%$ of men and women reported 

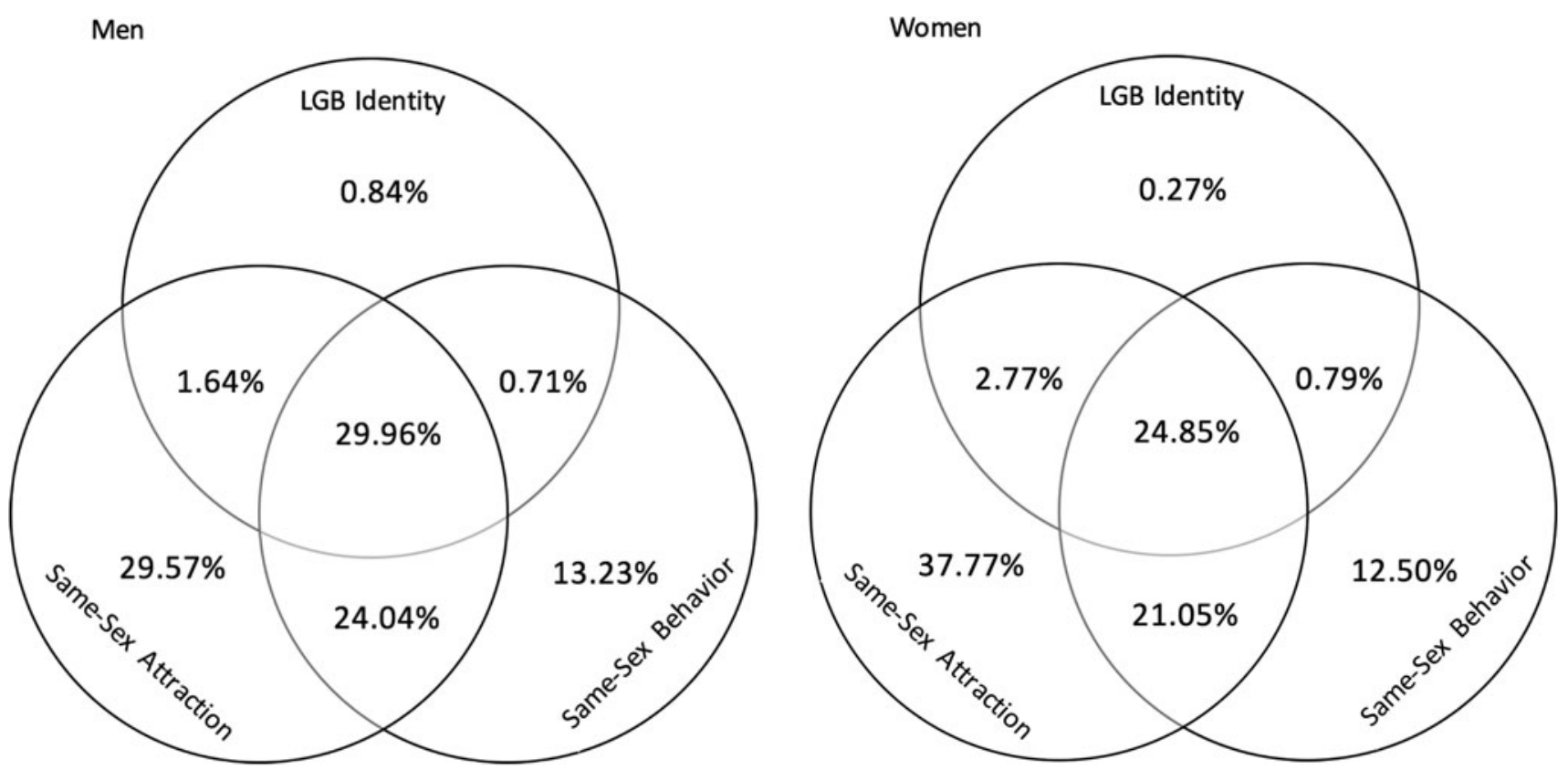

FIG. 1. Venn diagram of LGB identity, same-sex attraction, and same-sex behavior among sexual minority men and women in the National Epidemiologic Survey on Alcohol and Related Conditions-III. LGB, lesbian, gay, and bisexual.

an LGB identity in the absence of same-sex attraction or same-sex behavior.

\section{Differences among men}

Results from KHB logistic regression models testing the degree to which estimates change across unconditional and conditional models are displayed in Table 2; the corresponding $p$ value for $\triangle \mathrm{aOR}$ (adjusted odds ratio) signifies whether the change in the coefficient was statistically significant. Unconditional models testing sexual orientation differences in mental health and substance use disorders based on sexual attraction and behavior showed that exclusively same-sexattracted men had higher odds of major depressive disorder $(\mathrm{aOR}=1.84,95 \%$ confidence interval $[\mathrm{CI}]: 1.29-2.62)$ and a past-year suicide attempt $(\mathrm{aOR}=3.22$, 95\% CI: 1.04 9.95) than exclusively other-sex-attracted men, but were equally likely to meet the criteria for alcohol use disorder and tobacco use disorder. Men with sexual attractions to both men and women did not differ from men who reported exclusively other-sex attraction on any health outcome. Men who reported sexual behavior with both men and women, relative to men with exclusively other-sex behavior, had elevated odds of major depressive disorder $(\mathrm{aOR}=2.14,95 \%$ CI: 1.56-2.92), a past-year suicide attempt $(\mathrm{aOR}=4.15$, 95\% CI: $1.33-12.96)$, alcohol use disorder $(\mathrm{aOR}=1.54$, 95\% CI: 1.16-2.05), and tobacco use disorder $(\mathrm{aOR}=2.07,95 \% \mathrm{CI}: 1.62-2.64)$. Men who reported exclusively same-sex behavior indicated elevated risk for major depressive disorder $(\mathrm{aOR}=1.69,95 \% \mathrm{CI}: 1.19-2.39)$.

Results from the KHB comparison showed that all estimates of attraction- and behavior-based disparities in mental health and substance use disorders differed statistically across conditional and unconditional models, except for major depressive disorder and tobacco use disorder for men with exclusively same-sex behavior and those who reported sexual behavior with both men and women. For example, compared with unconditional models, there were no differences in major depressive disorder $(\mathrm{aOR}=1.39,95 \% \mathrm{CI}$ : 0.88-2.19) and past-year suicide attempts $(\mathrm{aOR}=1.14$, 95\% CI: 0.35-3.69) between exclusively same-sex-attracted and other-sex-attracted men after adjusting for sexual identity. Unexpectedly, conditional models revealed SM and sexual majority differences that were not present in unconditional models. For example, exclusively same-sex-attracted men were at lower risk for tobacco use disorder than exclusively other-sex-attracted men after adjusting for sexual identity $(\mathrm{aOR}=0.61,95 \% \mathrm{CI}: 0.46-0.81)$, suggesting a potential suppression effect.

\section{Differences among women}

Unconditional models showed that compared with exclusively other-sex-attracted and behavioral women, women who reported sexual attractions to and behaviors with both men and women had greater odds of major depressive disorder $(\mathrm{aOR}=1.78,95 \% \mathrm{CI}: 1.50-2.12$ and $\mathrm{aOR}=1.75,95 \%$ CI: $1.45-2.12$, respectively), past-year suicide attempt $(\mathrm{aOR}=2.39,95 \% \mathrm{CI}: 1.31-4.36$ and $\mathrm{aOR}=5.07,95 \% \mathrm{CI}$ : 3.08-8.34, respectively), alcohol use disorder $(\mathrm{aOR}=2.24$, 95\% CI: $1.94-2.58$ and $\mathrm{aOR}=2.55,95 \% \mathrm{CI}: 2.12-3.08$, respectively), and tobacco use disorder $(\mathrm{aOR}=1.57,95 \% \mathrm{CI}$ : $1.31-1.88$ and $\mathrm{aOR}=2.45,95 \% \mathrm{CI}: 2.02-2.98$, respectively). Exclusively same-sex-attracted women also had greater odds of alcohol use disorder ( $\mathrm{aOR}=2.29,95 \% \mathrm{CI}: 1.74-3.00)$ and tobacco use disorder $(\mathrm{aOR}=1.29,95 \% \mathrm{CI}: 1.02-1.65)$, and women who reported exclusively same-sex behavior were at greater odds of meeting the criteria for alcohol use disorder $(\mathrm{aOR}=2.05,95 \% \mathrm{CI}: 1.48-2.84)$, but not tobacco use disorder. 


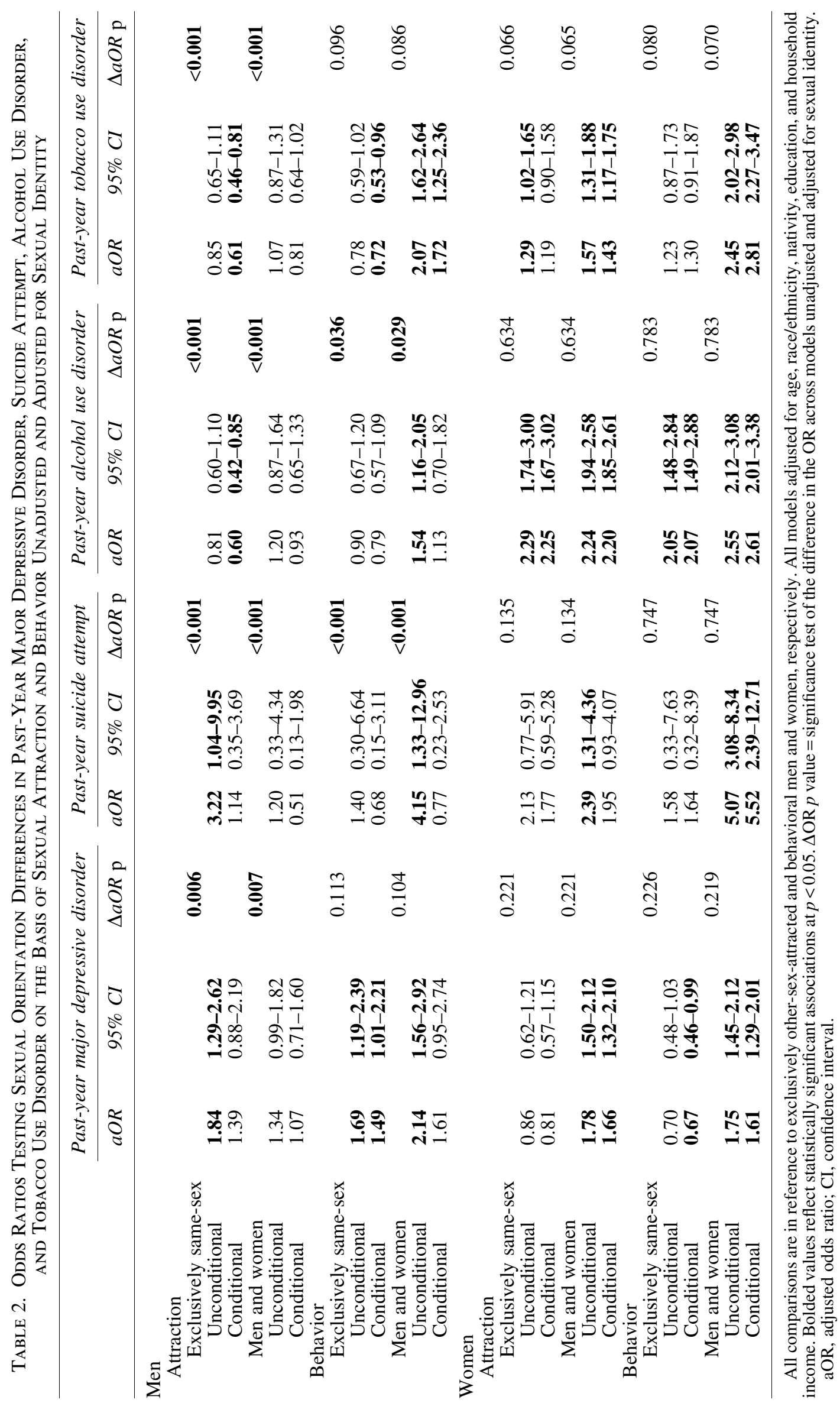


There were practical, but no statistical differences in the estimates for attraction- and behavior-based sexual orientation disparities in mental health and substance use disorders between unconditional and conditional models. For most outcomes, the inclusion of sexual identity mitigated sexual orientation differences in mental health and substance use disorders based on sexual attraction. The inclusion of sexual identity as a covariate attenuated SM and sexual majority differences in past-year suicide attempt for women attracted to both men and women $(\mathrm{aOR}=1.95,95 \% \mathrm{CI}$ : 0.93-4.07), and tobacco use disorder for exclusively samesex-attracted women $(\mathrm{aOR}=1.19,95 \% \mathrm{CI}$ : 0.90-1.58). Similar to men, however, estimates conditional on the inclusion of sexual identity revealed SM and sexual majority group differences that were not present in unconditional models. For example, women with exclusively same-sex sexual behavior had lower odds of major depressive disorder relative to women with exclusively other-sex sexual behavior after adjusting for sexual identity $(\mathrm{aOR}=0.67,95 \% \mathrm{CI}$ : 0.46-0.99). Notably, behavior-based SM and sexual majority differences in a past-year suicide attempt, past-year alcohol use disorder, and past-year tobacco use disorder were wider for women reporting sexual behavior with both men and women in conditional models, relative to unconditional models.

\section{Discussion}

In the current study, we propose a new approach to estimating sexual orientation-related disparities across multiple measures of sexual orientation. We contend that researchers should adjust attraction- and behavior-based disparity models to account for the unique and shared variance of sexual identity on the associations between attraction/behavior and health. That is, models testing associations between sexual attraction or behavior and health that adjust for sexual identity provide more accurate estimates of these associations independent of the influence of sexual identity; the association of which is already captured in models testing the relationship between sexual identity and health. Thus, analytic approaches to modeling health differences across sexual attraction, behavior, and identity are more readily attributable to these unique factors and comparable, given that they do not inadvertently replicate the statistical relationship between sexual identity and health across all three models. This approach is largely in response to the growing number of studies that estimate and compare sexual orientationrelated health disparities across multiple measures of sexual orientation. Our findings, we believe, reflect that this approach increases the specificity of results and the inferences and implications drawn from them.

Although the underlying argument for this approach is grounded in theoretical and empirical understandings of SM health inequities, ${ }^{22,23}$ we illustrate the utility of this approach using nationally representative data that have been used to provide some of the more prominent findings regarding sexual orientation disparities in mental health and substance abuse. ${ }^{8,10,17-19}$ Similar to previous research, $3,4,8,10$ our findings from unconditional models-models that do not adjust for sexual identity-showed different patterns of mental health and substance use disorders risk, across measures of sexual orientation, for both men and women. However, findings from conditional models-models adjusted for sexual identity-demonstrate the degree to which sexual identity plays both a unique and overlapping role in the relationship between sexual attraction/behavior and health, particularly among men. This sex difference may signify that sexual identity confers differential experiences that may increase risk for factors associated with health vulnerabilities for SM men relative to SM women. For example, SM men are more likely to experience identity-based victimization and discrimination relative to SM women, ${ }^{26}$ which may help to explain why sexual identity may be more likely to attenuate attraction- and behavior-based models for SM men. Ultimately, our findings suggest that estimating attractionand behavior-based differences in health independent of sexual identity either exaggerate or underestimate sexual orientation-related health disparities based on these characteristics.

Minority stress theory, which helps to explain the pathways through which SM-related health inequities emerge, can be applied broadly to SM populations based on sexual identity, attraction, or behavior. This is because all SM people likely experience some form of exposure to minority stressors (e.g., stigma, discrimination, and anticipated rejection), regardless of whether they adopt a SM identity. ${ }^{40,41}$ However, those who identify as LGB, or some other nonheterosexual sexual identity (e.g., queer), ascribe to a social identity that has experienced longstanding stigma and discriminatory practices. ${ }^{1,22}$ Preliminary evidence suggests that LGB-identified SM people are at greater risk for experiencing enacted stigma (e.g., discrimination) relative to SM people who do not adopt and assert these identities. ${ }^{18,19,26}$

At the same time, research also suggests that those who identify with a SM identity find greater opportunity for LGB community connectedness, social support, and other health-promoting resources. ${ }^{15,33}$ Indeed, there were instances where sexual orientation differences were wider (e.g., greater odds of poorer health for SM relative to sexual majority people) in behavior-based models that adjusted for sexual identity. This suggests that having an LGB identity may be associated with (potentially) protective resourcesfuture research should work to better understand the mechanisms through which having a SM identity may buffer or exacerbate health risk for those who report same-sex behaviors but do not adopt a SM identity. From a methods standpoint, our results highlight that the current research practice of independently assessing risks based on identity, attraction, and behavior independent of one another does not adequately address this complexity and, subsequently, the real-world strategies to address the systems and processes that maintain SM health inequities.

Although not the focus of our article, our analytic approach highlights how different components of sexual orientation (i.e., identity, attraction, and behavior) contribute to health risk in different ways for men and women. For example, sexual identity appears to alter the attraction and behavior effects on health more strongly for SM men than for SM women-particularly for mental health. Although speculative, this may reflect differences in the degree to which men and women experience or perceive sexual stigma. ${ }^{42-44}$ Although there has been preliminary work in this area, ${ }^{15,17,21}$ further research is necessary to better understand how mechanisms and processes of sexual orientation-related 
health disparities may differ in the presence or absence of sexual identity and how this might differ by sex.

\section{Implications for future research}

As the field of SM health expands, it remains important that data sources continue to include multiple measures of sexual orientation. We must, however, also challenge ourselves to innovate our approaches to modeling and understanding how these distinct but overlapping facets of sexual orientation are related to health. As shown in Figure 1, 32\% of same-sex-attracted men and $28 \%$ of same-sex-attracted women identify as LGB. This demonstrates the potential for LGB-identified people to bias estimates in attraction- and behavior-based disparity models, particularly when we consider that people who identify as LGB are more likely to experience minority stress mechanisms that contribute to elevated health risk among SM people. ${ }^{18,19,26}$ This approach also enables researchers to make stronger statements as to how health systematically varies across SM subgroups defined by patterns of sexual identity, attraction, and/or behavior (e.g., same-sex-attracted individuals who do and do not identify as LGB) - an area of growing interest in the field of SM health with meaningful implications for prevention and intervention strategies. ${ }^{15,17,18,21}$

Attraction- and behavior-based health disparity models that adjust for the influence of sexual identity offer more specificity given that they isolate the unique variance of a given health outcome associated with same-sex attraction or behavior. Once these effects are isolated, researchers are better able to assess how specific causal mechanisms may play a unique role in health risk for those who have same-sex attraction and/or behavior, but not a SM identity. Without accounting for sexual identity, testing these mechanisms may yield results and conclusions that may be misattributed to mechanisms that are specific to those who identify as LGB (see Fish et al., ${ }^{18}$ Evans-Polce et al., ${ }^{19}$ and Krueger et al. ${ }^{21}$ ). This last point is critically important for the subsequent development of prevention and intervention programs that meet the heterogeneous needs of an often oversimplified population. Recent research testing the relationship between anti-LGB discrimination and health illustrates that the association is largely driven by SM people who identify as LGB. ${ }^{18,19}$ Yet, heterosexual-SM people are still at greater risk for poor health relative to heterosexual adults who report exclusive other-sex attractions and behaviors; therefore, there are likely other mechanisms that contribute to their compromised health (e.g., internalized stigma). ${ }^{45,46}$ This level of specificity in conceptualizing and analyzing sexual orientation-related health disparities is necessary for developing and delivering effective SM health promotion strategies.

Finally, it is worth noting that our approach to adjusted estimates of attraction- and behavior-based health disparities need not only be applied when investigating sexual orientation health differences across all three measures; the same underlying concept applies when assessing either attraction or behavior with a given outcome-identity still plays a role in the health-related outcomes and mechanisms for SM people. We also recognize that there may be some situations where a researcher may consider the importance of modeling all three measures together, to better understand how each-if not multicollinear-could uniquely contribute to understandings of SM health. ${ }^{47}$

\section{Limitations}

There are several limitations to note. The NESARC does not reflect the growing diversity of SM people (e.g., queer, pansexual). Furthermore, to simplify the empirical demonstration of our arguments, we also operationalized sexual identity to reflect either a SM (i.e., lesbian/gay and bisexual) or heterosexual identity. Yet, with a growing body of literature that demonstrates the differential experiences of monosexual and plurisexual SM people, it is likely that lesbian/gay vs. bisexual identity differentially alters the association between sexual attraction or behavior and health. ${ }^{21}$ These ideas should be explored in future research. Although the current study does not take a developmental approach to sexual orientation, it is also important to recognize that crosssectional measures do not capture the developmental and dynamic nature of sexuality, which can influence health risk. ${ }^{16}$ It may be beneficial to consider and test how this approach may translate to models that attempt to capture sexual fluidity and health risk over time. We also excluded participants who reported not being sexually active for two reasons: (1) this group reflects a small proportion of the participants in our study; and (2) we wanted to keep our example of this method as parsimonious as possible. Future studies, of youth in particular, will need to test how sexual identity may alter attraction- and behavior-based differences in health for SM people who do not report sexual partners.

\section{Conclusion}

Our findings highlight the importance of accounting for the unique and shared effects of sexual identity when estimating attraction- and behavior-based differences in health. We argue that attraction- and behavior-based models that adjust for sexual identity more accurately capture health risk for SM populations and thus will provide more focused policy and prevention strategies for improving the health of SM people.

\section{Disclaimer}

Any interpretations and opinions expressed herein are solely those of the authors and may not reflect those of the Centers for Disease Control and Prevention or the National Institutes of Health.

\section{Author Disclosure Statement}

No competing financial interests exist.

\section{Funding Information}

This work was supported, in part, by the University of Maryland Prevention Research Center cooperative agreement number U48DP006382 from the Centers for Disease Control and Prevention. Dr. Fish also acknowledges support from P2CHD041041, awarded to the Maryland Population Research Center, by the Eunice Kennedy Shriver National Institute of Child Health and Human Development and 
from F32AA023138, awarded to Dr. Fish by the National Institute on Alcohol Abuse and Alcoholism.

\section{References}

1. Institute of Medicine (US) Committee on Lesbian, Gay, Bisexual, and Transgender Health Issues and Research Gaps and Opportunities: The Health of Lesbian, Gay, Bisexual, and Transgender People: Building a Foundation for Better Understanding. Washington, DC: National Academies Press, 2011.

2. Lick DJ, Durso LE, Johnson KL: Minority stress and physical health among sexual minorities. Perspect Psychol Sci 2013;8:521-548.

3. Plöderl M, Tremblay P: Mental health of sexual minorities. A systematic review. Int Rev Psychiatry 2015;27:367-385.

4. Talley AE, Gilbert PA, Mitchell J, et al.: Addressing gaps on risk and resilience factors for alcohol use outcomes in sexual and gender minority populations. Drug Alcohol Rev 2016;35:484-493.

5. Lindley LL, Walsemann KM, Carter JW, Jr.: The association of sexual orientation measures with young adults' health-related outcomes. Am J Public Health 2012;102: 1177-1185.

6. Muñoz-Laboy MA: Beyond "MSM": Sexual desire among bisexually-active Latino Men in New York City. Sexualities 2004;7:55-80.

7. Young RM, Meyer IH: The trouble with "MSM" and "WSW": Erasure of the sexual-minority person in public health discourse. Am J Public Health 2005;95:1144-1149.

8. Bostwick WB, Boyd CJ, Hughes TL, McCabe SE: Dimensions of sexual orientation and the prevalence of mood and anxiety disorders in the United States. Am J Public Health 2010;100:468-475.

9. Fish JN, Hughes TL, Russell ST: Sexual identity differences in high-intensity binge drinking: Findings from a US national sample. Addiction 2018;113:749-758.

10. McCabe SE, Hughes TL, Bostwick WB, et al.: Sexual orientation, substance use behaviors and substance dependence in the United States. Addiction 2009;104:1333-1345.

11. Fish JN, Schulenberg JE, Russell ST: Sexual minority youth report high-intensity binge drinking: The critical role of school victimization. J Adolesc Health 2019;64:186-193.

12. Kann L, Olsen EO, McManus T, et al.: Sexual identity, sex of sexual contacts, and health-related behaviors among students in grades 9-12-United States and selected sites, 2015. MMWR Surveill Summ 2016;65:1-202.

13. Korchmaros JD, Powell C, Stevens S: Chasing sexual orientation: A comparison of commonly used single-indicator measures of sexual orientation. J Homosex 2013;60:596614.

14. Reback CJ, Larkins S: Maintaining a heterosexual identity: Sexual meanings among a sample of heterosexually identified men who have sex with men. Arch Sex Behav 2010;39: 766-773.

15. Krueger EA, Upchurch DM: Are sociodemographic, lifestyle, and psychosocial characteristics associated with sexual orientation group differences in mental health disparities? Results from a national population-based study. Soc Psychiatry Psychiatr Epidemiol 2019;54:755770.

16. Fish JN, Pasley K: Sexual (minority) trajectories, mental health, and alcohol use: A longitudinal study of youth as they transition to adulthood. J Youth Adolesc 2015;44: 1508-1527.
17. McCabe SE, Matthews AK, Lee JGL, et al.: Tobacco use and sexual orientation in a national cross-sectional study: Age, race/ethnicity, and sexual identity-attraction differences. Am J Prev Med 2018;54:736-745.

18. Fish JN, Rice CE, Lanza ST, Russell ST: Is young adulthood a critical period for suicidal behavior among sexual minorities? Results from a US national sample. Prev Sci 2019;20: 353-365.

19. Evans-Polce RJ, Veliz PT, Boyd CJ, et al.: Associations between sexual orientation discrimination and substance use disorders: Differences by age in US adults. Soc Psychiatry Psychiatr Epidemiol 2020;55:101-110.

20. Meyer IH: Prejudice, social stress, and mental health in lesbian, gay, and bisexual populations: Conceptual issues and research evidence. Psychol Bull 2003;129:674-697.

21. Krueger EA, Fish JN, Upchurch DM: Sexual orientation disparities in substance use: Investigating social stress mechanisms in a national sample. Am J Prev Med 2020; 58:59-68.

22. Meyer IH: Prejudice as stress: Conceptual and measurement problems. Am J Public Health 2003;93:262-265.

23. Hatzenbuehler ML: How does sexual minority stigma "Get under the skin"? A psychological mediation framework. Psychol Bull 2009;135:707-730.

24. Meyer IH: Minority stress and mental health in gay men. J Health Soc Behav 1995;36:38-56.

25. Hatzenbuehler ML: Structural stigma and health inequalities: Research evidence and implications for psychological science. Am Psychol 2016;71:742-751.

26. Rice CE, Fish JN, Russell ST, Lanza ST: Sexual minorityrelated discrimination across the life course: Findings from a national sample of adults in the United States. J Homosex 2019. [Epub ahead of print]; DOI: 10.1080/00918369.2019.1648083

27. D'Augelli AR, Grossman AH, Starks MT: Families of gay, lesbian, and bisexual youth: What do parents and siblings know and how do they react? J GLBT Fam Stud 2008;4: 95-115.

28. Russell ST, Toomey RB, Ryan C, Diaz RM: Being out at school: The implications for school victimization and young adult adjustment. Am J Orthopsychiatry 2014;84: 635-643.

29. Goldbach JT, Tanner-Smith EE, Bagwell M, Dunlap S: Minority stress and substance use in sexual minority adolescents: A meta-analysis. Prev Sci 2014;15:350-363.

30. Ryan C, Huebner D, Diaz RM, Sanchez J: Family rejection as a predictor of negative health outcomes in White and Latino lesbian, gay, and bisexual young adults. Pediatrics 2009;123:346-352.

31. Hammack PL, Meyer IH, Krueger EA, et al.: HIV testing and pre-exposure prophylaxis (PrEP) use, familiarity, and attitudes among gay and bisexual men in the United States: A national probability sample of three birth cohorts. PLoS One 2018;13:e0202806.

32. Carrillo H, Hoffman A: From MSM to heteroflexibilities: Non-exclusive straight male identities and their implications for HIV prevention and health promotion. Glob Public Health 2016;11:923-936.

33. Watson RJ, Fish JN, Allen A, Eaton L: Sexual identity disclosure and awareness of HIV prevention methods among black men who have sex with men. J Sex Res 2018;55: 975-983.

34. Silva TJ, Whaley RB: Bud-sex, dude-sex, and heteroflexible men: The relationship between straight identification and social attitudes in a nationally representative sample of 
men with same-sex attractions or sexual practices. Sociol Perspect 2018;61:426-443.

35. Grant BF, Chu A, Amsbary M, et al.: Source and Accuracy Statement. National Epidemiologic Survey on Alcohol and Related Conditions-III (NESARC-III). Rockville, MD: National Institute on Alcohol Abuse and Alcoholism, 2014.

36. American Psychiatric Association: Diagnostic and Statistical Manual of Mental Disorders (DSM-5 ${ }^{\circledR}$ ). Washington, DC: American Psychiatric Association Publishing, 2013.

37. Kohler U, Karlson KB, Holm A: Comparing coefficients of nested nonlinear probability models. Stata J 2011;11:420438.

38. Karlson KB, Holm A, Breen R: Total, direct, and indirect effects in logit models. Aarhus, Denmark: Centre for Strategic Research in Education, Aarhus University, 2010. Available at www.forskningsdatabasen.dk/en/catalog/2353728899 Accessed August 8, 2018.

39. Breen R, Holm A, Karlson KB: A reinterpretation of coefficients from logit, probit, and other non-linear probability models: Consequences for comparative sociological research. 2011. Available at https://papers.ssrn.com/sol3/ papers.cfm?abstract_id=1857431 Accessed August 10, 2018.

40. Cain R: Stigma management and gay identity development. Soc Work 1991;36:67-73.

41. Pachankis JE: The psychological implications of concealing a stigma: A cognitive-affective-behavioral model. Psychol Bull 2007;133:328-345.

42. Herek GM: Sexual prejudice and gender: Do heterosexuals' attitudes toward lesbians and gay men differ? J Soc Issues 2000;56:251-266.
43. Smith AM, Rissel CE, Richters J, et al.: Sex in Australia: Sexual identity, sexual attraction and sexual experience among a representative sample of adults. Aust N Z J Public Health 2003;27:138-145.

44. Purdie-Vaughns V, Eibach RP: Intersectional invisibility: The distinctive advantages and disadvantages of multiple subordinate-group identities. Sex Roles 2008;59:377-391.

45. Shoptaw S, Weiss RE, Munjas B, et al.: Homonegativity, substance use, sexual risk behaviors, and HIV status in poor and ethnic men who have sex with men in Los Angeles. J Urban Health 2009;86:77-92.

46. Vu L, Tun W, Sheehy M, Nel D: Levels and correlates of internalized homophobia among men who have sex with men in Pretoria, South Africa. AIDS Behav 2012;16:717723.

47. Matthews DD, Blosnich JR, Farmer GW, Adams BJ: Operational definitions of sexual orientation and estimates of adolescent health risk behaviors. LGBT Health 2014;1:42-49.

Address correspondence to: Jessica N. Fish, PhD Department of Family Science School of Public Health

University of Maryland 4200 Valley Drive, Suite 2242 College Park, MD 20742-2611 $U S A$

E-mail: jnfish@umd.edu 